\title{
Ammonia as a Temperature Tracer in the Ultraluminous Galaxy Merger Arp 220
}

\author{
Jürgen Ott \\ National Radio Astronomy Observatory, P.O. Box O, 1003 Lopezville Road, Socorro, NM \\ 87801, USA \\ jott@nrao.edu \\ Christian Henkel \\ Max-Planck-Institut für Radioastronomie, Auf dem Hügel 69, 53121 Bonn, Germany \\ Astronomy Department, Faculty of Science, King Abdulaziz University, P.O. Box 80203, \\ Jeddah, Saudi Arabia \\ chenkel@mpifr-bonn.mpg.de \\ James A. Braatz \\ National Radio Astronomy Observatory, 520 Edgemont Road, Charlottesville, VA 22903, \\ USA \\ jbraatz@nrao.edu \\ and \\ Axel Weiß \\ Max-Planck-Institut für Radioastronomie, Auf dem Hügel 69, 53121 Bonn, Germany \\ aweiss@mpifr-bonn.mpg.de
}

\begin{abstract}
We present Australia Telescope Compact Array (ATCA) and Robert C. Byrd Green Bank Telescope (GBT) observations of ammonia $\left(\mathrm{NH}_{3}\right)$ and the $1.2 \mathrm{~cm}$ radio continuum toward the ultraluminous infrared galaxy (ULIRG) merger Arp 220. We detect the $\mathrm{NH}_{3}(1,1),(2,2),(3,3),(4,4),(5,5)$, and $(6,6)$ inversion lines in absorption against the unresolved, $(62 \pm 9)$ mJy continuum source at $1.2 \mathrm{~cm}$. The peak apparent optical depths of the ammonia lines range from $\sim$
\end{abstract}


0.05 to 0.18 . The absorption lines are well described by single-component Gaussians with central velocities in between the velocities of the eastern and western cores of Arp 220. Therefore, the ammonia likely traces gas that encompasses both cores. The absorption depth of the $\mathrm{NH}_{3}(1,1)$ line is significantly shallower than expected based on the depths of the other transitions. The shallow $(1,1)$ profile may be caused by contamination from emission by a hypothetical, cold $(\lesssim 20 \mathrm{~K})$ gas layer with an estimated column density of $\lesssim 2 \times 10^{14} \mathrm{~cm}^{-2}$. This layer would have to be located behind or away from the radio continuum sources to produce the contaminating emission. The widths of the ammonia absorption lines are $\sim 120-430 \mathrm{~km} \mathrm{~s}^{-1}$, in agreement with those of other molecular tracers. We cannot confirm the extremely large linewidths of up to $\sim 1800 \mathrm{~km} \mathrm{~s}^{-1}$ previously reported for this galaxy. Using all of the ATCA detections except for the shallow $(1,1)$ line, we determine a rotational temperature of $(124 \pm 19) \mathrm{K}$, corresponding to a kinetic temperature of $T_{\text {kin }}=(186 \pm 55) \mathrm{K}$. Ammonia column densities depend on the excitation temperature. For excitation temperatures of $10 \mathrm{~K}$ and $50 \mathrm{~K}$, we estimate $N\left(\mathrm{NH}_{3}\right)=(1.7 \pm 0.1) \times 10^{16} \mathrm{~cm}^{-2}$ and $(8.4 \pm 0.5) \times 10^{16} \mathrm{~cm}^{-2}$, respectively. The relation scales linearly for possible higher excitation temperatures. Our observations are consistent with an ortho-to-para-ammonia ratio of unity, implying that the ammonia formation temperature exceeds $\sim 30 \mathrm{~K}$. In the context of a model with a molecular ring that connects the two nuclei in Arp 220, we estimate the $\mathrm{H}_{2}$ gas density to be $\sim f_{\mathrm{V}}^{-0.5} \times(1-4) \times 10^{3}$, where $f_{\mathrm{V}}$ is the volume filling factor of the molecular gas. In addition to ammonia, our ATCA data show an absorption feature adjacent in frequency to the $\mathrm{NH}_{3}(3,3)$ line. The line does not appear in the GBT spectrum. If we interpret the line to be from the $\mathrm{OH}^{2} \Pi_{3 / 2} J=9 / 2 F=4-4$ transition, it would have a linewidth, systemic velocity, and apparent optical depth similar to what we detect in the ammonia lines. Comparing the new line to the previously detected $6 \mathrm{GHz}^{\mathrm{OH}}{ }^{2} \Pi_{3 / 2} J=5 / 2$ $F=2-2$ transition, we determine a rotational $\mathrm{OH}$ temperature of $\sim 245 \mathrm{~K}$, about 2 times the rotational temperature of ammonia. If this association with $\mathrm{OH}$ is correct, it marks the first detection of the highly excited $(\sim 511 \mathrm{~K}$ above ground state) ${ }^{2} \Pi_{3 / 2} J=9 / 2 F=4-4 \mathrm{OH}$ line in an extragalactic object.

Subject headings: galaxies: individual (Arp 220) — galaxies: starburst — galaxies: ISM — galaxies: nuclei — ISM: molecules — radio lines: galaxies

Arp 220 


\section{Introduction}

Ultraluminous infrared galaxies (ULIRGs), with far-infrared luminosities exceeding $L_{\mathrm{FIR}} \gtrsim 10^{12} \mathrm{~L}_{\odot}$, exhibit some of the highest star formation (SF) rates encountered in the Universe (for a review, see Sanders \& Mirabel 1996). They are mergers containing at least two massive, gas-rich galaxies whose dust is heated during the merging process. Cloudcloud collisions and the subsequent inflow of gas feed nuclear activity (Nagar et al. 2003) and trigger an amazingly high rate of star formation in the central few $100 \mathrm{pc}$, typically reaching tens to hundreds of $\mathrm{M}_{\odot} \mathrm{yr}^{-1}$ (e.g., Flores et al. 2004). ULIRGs are particularly important galaxies as they are the most readily detectable population for which the SF per co-moving volume can be traced out to high redshifts (e.g., Madau et al. 1998; Cole et al. 2001; Darling \& Giovanelli 2002). Furthermore, the number of luminous IR galaxies per co-moving volume was much greater at high redshift, so these galaxies dominated the production of stars early in the Universe (e.g. Bridge et al. 2007; Hopkins et al. 2010). There is increasing evidence that ULIRGs may enter an optically bright quasar phase subsequently in the merging process (e.g. Hutchings \& Neff 1988; Canalizo \& Stockton 2001; Dasvra et al. 2007; Veilleux et al. 2009).

The prototypical ULIRG is $\operatorname{Arp} 220\left(\right.$ IC $\left.4553 ; L_{\mathrm{FIR}} \sim 1.5 \times 10^{12} L_{\odot}\right)$, at a distance of only $D \sim 75 \mathrm{Mpc}$. Its proximity allows us to study physical processes in more detail than we can for its more distant cousins. Arp 220 has two starburst nuclei (e.g., Norris 1988; Downes \& Solomon 1998) separated by 0.95 (350 pc) at a position angle of $92^{\circ}$. Many molecules and associated tracers have been detected in Arp 220, including CO,C I, C II, CS, $\mathrm{HCN}, \mathrm{HNC}, \mathrm{HCO}^{+}, \mathrm{H}_{2} \mathrm{CO}, \mathrm{H}_{2} \mathrm{O}, \mathrm{H}_{2} \mathrm{~S}, \mathrm{HC}_{3} \mathrm{~N}, \mathrm{HNCO}$, and thermal OH(e.g., Solomon et al. 1990, 1992; Hüttemeister et al. 1995a; Scoville. Yun. \& Brvant 1997; Solomon et al. 1997; Gerin \& Phillips 1998; Papadopoulos \& Seaquist 1998; Yao et al. 2003; Arava et al. 2004; Downes \& Eckart 2007; Salter et al. 2008; Aalto et al. 2009; Greve et al. 2009; Martín et al. 2011) as well as OH megamaser emission (e.g., Baan et al. 1982; Rovilos et al. 2003). The molecular gas traced by (sub-)arcsecond CO imaging reveals small, dense disks around each of the two nuclei. These small disks are embedded in a ring or disk that encompasses both cores, and that ring is itself embedded in an even larger molecular disk, $\sim 2.5 \mathrm{kpc}$ in size and with a density of $\sim 10^{2} \mathrm{~cm}^{-3}$ (Scoville, Yun, \& Bryant 1997; Downes \& Solomon 1998; Sakamoto et al. 1999; Mundell et al. 2001). The western nucleus has a higher molecular mass than its eastern counterpart, and may contain a supermassive black hole (Downes \& Eckart 2007). Both nuclei show signatures of outflows (Sakamoto et al. 2009). Direct measurements of the physical properties of the gas, however, are difficult. For linear molecules, radiative transfer models, like the Large Velocity Gradient (LVG) analysis, have solutions that are degenerate between high temperature/low density and low temperature/high density. Anal-

ysis of the excitation conditions show that the two cores have temperatures in the range 
$\sim 45-120 \mathrm{~K}$ and densities of order $10^{6} \mathrm{~cm}^{-3}$, with the western nucleus being somewhat warmer and denser (e.g. Aalto et al. 2009; Greve et al. 2009; Matsushita et al. 2009). Farther out, while the gas is known to be warm $(T \gtrsim 40 \mathrm{~K})$, no accurate temperature or density measurements exist.

Solomon et al. (1992) and Gao \& Solomon (2004a, b) observed CO and HCN in a large sample of ULIRGs. They conclude that HCN is a much better tracer of SF than CO, and in fact they find that the HCN line luminosity scales linearly with the SF rate in galaxies. HCN exhibits a large electric dipole moment and the molecule is only excited when the gas is sufficiently dense $\left(n\left(\mathrm{H}_{2}\right) \gtrsim 10^{4} \mathrm{~cm}^{-3}\right)$. Dense molecular gas is therefore the best tracer for the material that is readily converted into stars (but see Graciá-Carpio et al. 2006, for caveats).

Similar to $\mathrm{HCN}$, ammonia $\left(\mathrm{NH}_{3}\right)$ also traces dense gas, though it is excited at densities down to $\sim 10^{3-4} \mathrm{~cm}^{-3}$. Its abundance is closely linked to that of HCN by the cyanide chemistry network (e.g., Schilke et al. 1992). In contrast to linear molecules like CO and HCN, the ammonia molecule is a prolate symmetric top with tetrahedral structure. Most of its excitation states, characterized by the quantum numbers $(J, K)$, show 'inversion doublets' caused by the nitrogen atom tunneling through the plane defined by the three hydrogen atoms. The lowest inversion doublet of each $K$-ladder (with the quantum numbers $J=K$ ) is metastable. The relative populations of the metastable doublets are mainly determined by collisions, follow a Boltzmann distribution, and thus provide one of the most suitable thermometers to estimate kinetic temperatures of extragalactic molecular gas (e.g., Walmslev \& Ungerechts 1983; Ungerechts, Winnewisser, \& Walmsley 1986; Danby et al. 1988; Henkel et al. 2000). Since the energy separation between the individual inversion doublets is only a weak function of $J$ and $K$, a vast range of excitation properties can be covered by observations of lines closely spaced in frequency. The most relevant $\mathrm{NH}_{3}$ lines fall in the $1.2 \mathrm{~cm}$ band $(\nu \sim 25 \mathrm{GHz})$. Relative calibration of these lines tends to be very good since they can be observed nearly simultaneously, under similar atmospheric conditions, and with the same telescope, receiver, and backend.

$\mathrm{NH}_{3}$ has been detected in Arp 220 by Takano et al. (2005) with the $45 \mathrm{~m}$ Nobeyama single dish telescope. Their main result was that the ammonia has linewidths of $\sim 1800 \mathrm{~km} \mathrm{~s}^{-1}$, which they suggest could indicate that the gas is located in an accretion disk around an active nucleus. Alternatively, the wide lines may indicate strong molecular inflows or outflows. Single dish observations, however, can be affected by instabilities that lead to poor spectral baselines and uncertain line profiles. This is particularly the case for broad lines. Here, we present ATCA interferometric data with higher signal to noise and better spectral baselines. We also present a sensitive GBT spectrum. In Sect.2 we describe our observational setups. 
We present our results in Sect.3, along with a critical comparison of our data with those of Takano et al. (2005). In Sect.4 we derive and interpret the apparent optical depths, gas temperatures, gas densities and kinematics of Arp 220, as well as its SF properties. Sect.5 summarizes our paper.

\section{Observations and Data Reduction}

\subsection{ATCA Observations}

We observed the spectral lines of ammonia and the $1.2 \mathrm{~cm}$ (K-band) continuum toward Arp 220 with the ATCA 1 . The data were taken in several stages: (1) A broadband spectrum was obtained covering the $23.2-23.6 \mathrm{GHz}$ frequency range. At the time of the observations (2005 July), the ATCA could not cover this full frequency range in a single observation. Therefore, we configured the observations with $128 \mathrm{MHz}$ bands of 64 channels each, and stepped through the desired frequency range in $60 \mathrm{MHz}$ increments (indicated by the bars at the bottom of Fig.11), with two bands observed at a time. The observations were performed over 4 days with $\sim 4.5 \mathrm{~h}$ on-source per day. The spectral resolution is equivalent to 2.2 channels $\left(\sim 57 \mathrm{~km} \mathrm{~s}^{-1}\right)$. Calibration was performed individually for each frequency band and observing day. At the time, the ATCA was in its most compact H 75 antenna configuration, where baselines reach from $31 \mathrm{~m}$ to $89 \mathrm{~m}$. (2) The $(6,6)$ line was observed in 2005 March with the H 214 antenna configuration, which has $82 \mathrm{~m}-247 \mathrm{~m}$ baselines. The integration times on source were $\sim 2.5 \mathrm{~h}$ (2005 March 17), and $\sim 4.5 \mathrm{~h}$ (2005 March 20). (3) On 2005 July 30 we observed the $(4,4)$ line on source for $4.5 \mathrm{~h}$ in the $\mathrm{H} 75$ configuration. (4) A day later we measured the $(5,5)$ line with the same integration time in the same array configuration. The correlator settings for stages (2)-(4) were the same as in stage (1), except that, for the $(4,4)$ and $(5,5)$ transitions, only two $128 \mathrm{MHz}$ wide frequency settings were used in parallel and separated by $72 \mathrm{MHz}$. The $(6,6)$ line was observed in a single frequency window. A summary of the observational setups is provided in Table1.

The data were processed using the MIRIAD software package. We observed PKS 1253055 for bandpass calibration, PKS 1934-638 for flux calibration, and PKS 1538+149 (in 20 min intervals) for phase/gain calibration. For each spectrum, we subtracted the continuum as determined by a linear fit to the line-free channels. In the process we also created continuum data sets. All data were Fourier transformed using 'natural' weighting. To obtain

\footnotetext{
${ }^{1}$ The Australia Telescope Compact Array is part of the Australia Telescope which is funded by the Commonwealth of Australia for operation as a National Facility managed by CSIRO.
} 
the spectra, the data were not deconvolved due to the lack of spatial structure on the scales of the dirty beam (source size $\ll 10^{\prime \prime}$ versus $\sim 10^{\prime \prime}-40^{\prime \prime}$ beam size, the latter depending on the array configuration, see Table1). Deconvolution with CLEAN is only needed to reconstruct source morphologies. This, however, comes for the prize of introducing non-linearities. For our unresolved source, we decided not to CLEAN the data. Given the relatively strong continuum flux, this implies that the noise within the planes of the dirty images is dominated by sidelobes and we measure $5-10 \mathrm{mJy}$ beam $^{-1}$ depending on the observations. For an estimate of the more representative statistical noise and thus for the apparent optical depth uncertainty, however, we did apply CLEAN deconvolution to remove the sidelobes. This method yields rms values of $\sim 0.4-1.2 \mathrm{mJy}$ beam $^{-1}$ per channel for the different observations. We estimate the absolute calibration uncertainties to be of order $15 \%$.

\subsection{GBT Observations}

We also observed the $1.2 \mathrm{~cm}$ ammonia spectrum toward Arp 220 on 2006 January 21 and 2006 February 02 with the Green Bank Telescope (GBT) of the National Radio Astronomy Observatory 2 (see Table1). We observed both circular polarizations simultaneously using a total-power nodding mode in which the target position was placed alternately every $2.5 \mathrm{~min}$ in one of the two beams of the $22-26 \mathrm{GHz}$ K-band receiver. The beams are spaced by $3^{\prime}$ in the cross-elevation direction. Total time on source was 40 minutes. We configured the spectrometer with an $800 \mathrm{MHz}$ spectral window centered on the $\mathrm{NH}_{3}(3,3)$ line for $\operatorname{Arp} 220$. The data were calibrated and averaged in GBTIDL. During calibration, we used an estimate of the atmospheric opacity obtained from weather conditions. We boxcar averaged the spectrum to achieve $1.56 \mathrm{MHz}$ channel spacing, and subtracted a fifth-order polynomial fit to the line-free channels over the full $800 \mathrm{MHz}$, a conservative approach for broadband GBT data. Finally we combined the spectra from the two days and the rms noise of the final data is $0.8 \mathrm{mJy}$ per $1.56 \mathrm{MHz}$ channel.

\footnotetext{
${ }^{2}$ The National Radio Astronomy Observatory is a facility of the National Science Foundation operated under cooperative agreement by Associated Universities, Inc.
} 


\section{Results}

\section{1. $\quad 1.2 \mathrm{~cm}$ Continuum Emission}

With the ATCA, we detect an unresolved continuum source toward Arp 220 at RA (J2000) $=15^{h} 34^{m} 57.2^{s}$, DEC $(\mathrm{J} 2000)=23^{\circ} 30^{\prime} 11^{\prime \prime}$. The source has a flux density at $23.4 \mathrm{GHz}$ of $208 \mathrm{mK}$ or $62 \mathrm{mJy}$ (error of order $15 \%$ ) measured within the $31.1^{\prime \prime} \times 21.1^{\prime \prime}$ beam. From March to August 2005, the measured continuum flux varied by less than $5 \%$.

\subsection{Ammonia Inversion Lines}

In Figs.11and 2 we show ATCA spectra of Arp 220 in units of apparent optical depth (see Sect.4.1), and Fig. 3 shows a GBT spectrum overlaid on the ATCA data. All six observed ammonia inversion lines, $\mathrm{NH}_{3}(J, K)=(1,1),(2,2),(3,3),(4,4),(5,5)$, and $(6,6)$, are detected in absorption against the radio continuum of Arp 220. We do not detect clear emission features, but the broadband ATCA spectrum displays slightly positive values adjacent to the $(1,1)$ absorption line (see Sects.4.1 and 4.2 for a discussion). We fitted single-component Gaussian profiles to all observed absorption lines. The resulting apparent optical depths $\tau$ (see Sec.4.1 for their derivation), the optical, barycentric peak velocities $v_{\mathrm{opt} \text {,bary }}^{\text {peak }}$, the linewidths $\Delta v_{1 / 2}$, and the apparent optical depths integrated over the line profiles, $\int \tau \mathrm{d} v$, are given in Table 2 and the fits are shown in Fig2, The fitted line centroids are all within $\sim 60 \mathrm{~km} \mathrm{~s}^{-1}$ of the systemic velocity of $5434 \mathrm{~km} \mathrm{~s}^{-1}(z=0.018126)$. This is remarkable given the $\sim 57 \mathrm{~km} \mathrm{~s}^{-1}$ velocity resolution of the ATCA observations (see Sect.2). The linewidths range between $\sim 230 \mathrm{~km} \mathrm{~s}^{-1}$ and $\sim 330 \mathrm{~km} \mathrm{~s}^{-1}$ for most lines. The $(1,1)$ and $(3,3)$ lines, however, have widths of $121 \pm 38 \mathrm{~km} \mathrm{~s}^{-1}$ and $435 \pm 19 \mathrm{~km} \mathrm{~s}^{-1}$, more extreme than the other transitions. Note, though, that the $(3,3)$ line slightly overlaps with another absorption feature, described in Sect.3.3. Our fitted linewidths are in agreement with those found for other molecular tracers (e.g. Salter et al. 2008) but contradict the results presented in Takano et al. (2005) for the $\mathrm{NH}_{3}(1,1)$ and $(3,3)$ lines. We cannot confirm the extremely wide lines of up to $\sim 1800 \mathrm{~km} \mathrm{~s}^{-1}$ observed by Takano et al. (2005) with the Nobeyama $45 \mathrm{~m}$ telescope. None of the lines measured with the ATCA or GBT have widths exceeding $\sim 450 \mathrm{~km} \mathrm{~s}^{-1}$ (see Table2 and Figs.113).

There are some notable differences in widths and strengths of the lines measured with the GBT and the ATCA. The reason may be the weakness of the lines, the complexity of the spectra, and potential confusion with the continuum emission from Arp220. The $\mathrm{NH}_{3}(1,1)$ and $(2,2)$ lines are separated by less than $30 \mathrm{MHz}$ so, for the GBT data, it is difficult to precisely define a spectral baseline near these features. The quality of spectral 
baselines from the interferometric ATCA data, on the other hand, is very good. We would like to add, however, that regridding the $128 \mathrm{MHz}$ blocks of our ATCA observations into a single spectrum conveys some uncertainty. For the relatively isolated $\mathrm{NH}_{3}(4,4)$ line, our results agree well with those of Takano et al. (2005). The centroids of the Gaussian line fits between the GBT and ATCA also differ by a few tens of $\mathrm{km} \mathrm{s}^{-1}$ (Table2). This may be caused by the limited signal-to-noise ratios of the spectra, despite the relatively small errors of the Gaussian fits. Gaussian fitting may have small systematic errors caused by the uncertain baseline subtraction and blended lines. The linewidths and peak apparent optical depths of the $\mathrm{NH}_{3}(1,1)$ line agree between the ATCA and GBT observations. The other lines, however, disagree to some extent in linewidth and peak apparent optical depth. The $(3,3)$ line is about twice as broad in the ATCA data as in the GBT data and the peak apparent optical depths in the GBT data for the $\mathrm{NH}_{3}(2,2)$ and $(3,3)$ lines are about $1 / 3$ less than those in the ATCA spectrum.

\subsection{Potential Detection of $\mathrm{OH}^{2} \Pi_{3 / 2} J=9 / 2$}

In addition to the $(1,1)$ through $(6,6)$ ammonia absorption lines, the broadband ATCA spectrum exhibits an additional absorption feature at $\sim 23.39 \mathrm{GHz}$ (Fig.11). As with the ammonia lines, this additional spectral line appears in both spectra that were taken at that frequency (the horizontal bars in Fig.1 show the tunings of the individual observations). Note, however, that the two underlying individual spectra were observed simultaneously which implies that they are subject to a common systematic uncertainty and are not entirely independent measurements. At the redshift of Arp 220, the most obvious line candidates would be the $F=4-4$ and $F=5-5$ doublet of the $\mathrm{OH}^{2} \Pi_{3 / 2} J=9 / 2$ transition at rest frequencies of $23817.6153 \mathrm{MHz}$ and $23826.6211 \mathrm{MHz}$, respectively 3 . This doublet is at an energy $\sim 511 \mathrm{~K}$ above ground state and indicates highly excited gas. Adopting the rest frequency of the $\mathrm{F}=4-4$ transition and fitting a Gaussian to this line, we get a line center of $-13 \pm 10 \mathrm{~km} \mathrm{~s}^{-1}$ (relative to the systemic velocity of $5434 \mathrm{~km} \mathrm{~s}^{-1}$ ) and a linewidth of $292 \pm 29 \mathrm{~km} \mathrm{~s}^{-1}$, very similar to the ammonia lines. These fitting results are also shown in Table2, Fitting the $\mathrm{F}=5-5$ line alone, we find the centroid of the Gaussian would be offset by $\sim+100 \mathrm{~km} \mathrm{~s}^{-1}$ from systemic, likely too large for a feature that belongs to Arp 220. Since the doublet lines are spaced by only $\sim 113 \mathrm{~km} \mathrm{~s}^{-1}$, however, they may be blended to produce the observed line width. A fit from blended lines would have a mean velocity offset of $\sim+44 \mathrm{~km} \mathrm{~s}^{-1}-$ well within the range of the line velocities observed. In either case, if the measured absorption feature indeed corresponds to one or both $\mathrm{OH}^{2} \Pi_{3 / 2} J=9 / 2$ lines, it would be the first

\footnotetext{
${ }^{3}$ taken from the LOVAS catalog: http://physics.nist.gov/cgi-bin/micro/table5/start.pl
} 
detection of this transition in an extragalactic object. To date, this doublet has only been observed toward Galactic ultracompact H II regions such as W3(OH) (e.g. Winnberg et al. 1978; Wilson et al. 1991; Baudry \& Menten 1995).

Lower energy $\mathrm{OH}$ doublets have been observed previously in Arp 220. These include ${ }^{2} \Pi_{1 / 2} J=1 / 2$ at $4.7 \mathrm{GHz},{ }^{2} \Pi_{3 / 2} J=5 / 2$ at $6.0 \mathrm{GHz}$, and ${ }^{2} \Pi_{1 / 2} J=3 / 2$ at $7.8 \mathrm{GHz}$ (Henkel et al. 1986, 1987; Salter et al. 2008). These detections show offset velocities in the range $-20 \mathrm{~km} \mathrm{~s}^{-1}$ to $+5 \mathrm{~km} \mathrm{~s}^{-1}$, linewidths of $\sim 250-480 \mathrm{~km} \mathrm{~s}^{-1}$, and apparent optical depths of $\tau \sim 0.05-0.11$. The parameters of these lines are consistent with those of our possible ATCA OH line detection. Using our possible line and the Salter et al. $2008{ }^{2} \Pi_{3 / 2} J=5 / 2$ $\mathrm{F}=2-2$ detection, we estimate a rotational temperature of $\sim 245 \mathrm{~K}$, assuming Local Thermal Equilibrium. This estimate is about a factor of two higher than the rotational temperature we determine for ammonia (Sect.4.2). The line could be verified with wideband interferometric observations, for example with the new CABB backend of the ATCA or WIDAR on the EVLA. Indirect evidence could come from HERSCHEL data, via observations of rotational lines between different $\mathrm{OH}$ doublets (e.g. Wampfler et al. 2010).

\section{Discussion}

\subsection{Apparent Optical Depths}

For a molecular cloud that partly covers a continuum source, in the Rayleigh-Jeans limit, we can express the measured brightness temperature of a spectral line $\left(T_{\mathrm{L}}\right)$ as:

$$
T_{\mathrm{L}}=\left(f_{\mathrm{cl}} T_{\mathrm{ex}}-f_{0} f_{\mathrm{C}} T_{\mathrm{C}}-f_{\mathrm{cl}} T_{\mathrm{CMB}}\right) \times\left(1-e^{-\tau}\right)
$$

(Rohlfs \& Wilson 2004) where $T_{\mathrm{ex}}$ is the excitation temperature of the line, $T_{\mathrm{C}}$ is the brightness temperature of a background continuum source, $T_{\mathrm{CMB}}$ is the brightness temperature of the cosmic microwave background, and $\tau$ is the optical depth of the line. The temperatures are scaled by the beam filling factors of the cloud $\left(f_{\mathrm{cl}}\right)$ and the continuum source $\left(f_{\mathrm{C}}\right)$. The source covering factor $f_{0}$ describes the fraction of the continuum covered by the cloud along the line of sight. We use this equation to determine the apparent optical depth $\tau$. The source covering factor $f_{0}$ can be assumed to be close to unity since the individual radio continuum emitting regions are relatively small, at most a few tens of pc in size (Rovilos et al. 2003; Lonsdale et al. 2006; Parra et al. 2007), and supposedly contain mostly supernova remnants embedded in the molecular gas (see Sect.4.4). In addition, the mean gas column density in the model of Downes \& Solomon (1998) is $N_{\mathrm{H}_{2}} \sim 2.5 \times 10^{24} \mathrm{~cm}^{-2}$ and the mean gas volume 
density is $\sim 500 \mathrm{~cm}^{-3}$ (using the radius of the outer molecular disk of $1.2 \mathrm{kpc}$, which corresponds for a cylindrical, edge-on geometry to a mean width and line of sight of $1.6 \mathrm{kpc}$, a height of the cylinder of $80 \mathrm{pc}$, and a gas mass of $5 \times 10^{9} \mathrm{M}_{\odot}$; but see Sect.4.3). Those large densities indicate that molecular gas is abundant and, closer to the starburst cores, ammonia is expected to be present over large regions which justifies our assumption of a source covering factor near unity. Under these circumstances we can also assume that both the cloud and the continuum filling factors are the same for absorption lines, i.e., $f_{\mathrm{cl}}=f_{\mathrm{C}}$. Ammonia not along the line of sight toward the radio continuum would appear in emission, which is not directly observed (but note the weak, tentative emission features adjacent to the $\mathrm{NH}_{3}(1,1)$ line [Fig.1] - the peculiarity of the $(1,1)$ transition is discussed in Sect.4.2). Under these conditions, Eq.1 simplifies to

$$
T_{\mathrm{L}}=f_{\mathrm{C}}\left(T_{\mathrm{ex}}-T_{\mathrm{C}}-T_{\mathrm{CMB}}\right) \times\left(1-e^{-\tau}\right) .
$$

VLBI continuum measurements at $18 \mathrm{~cm}$ show that the radio continuum of Arp 220 has an extent of $\lesssim 0.6 \square^{\prime \prime}$ (Smith et al. 1998; Rovilos et al. 2003), which corresponds to a beam filling factor of $f_{\mathrm{C}} \lesssim 10^{-3}$ when compared to the beam of our ATCA broadband observations $\left(\sim 31^{\prime \prime} .1 \times 21^{\prime \prime} .1\right)$. For this value, the measured Arp $2201.2 \mathrm{~cm}$ continuum brightness temperature of $T_{\mathrm{C}}^{\prime}=f_{\mathrm{C}} T_{\mathrm{C}}=208 \mathrm{mK}$ then implies a true brightness temperature $T_{\mathrm{C}} \gtrsim 208 \mathrm{~K}$. The best spatial resolution was obtained for the $\mathrm{NH}_{3}(6,6)$ line (Sect.2.1) and even for the $\sim 8$ times (in area) smaller beam of that observation we do not detect any extent of the continuum source. For this smaller beam of the $\mathrm{NH}_{3}(6,6)$ observation, the same approximation then boosts the true continuum temperature limit by the same factor of 8 . On the other hand, VLBI does filter out flux on larger scales which somewhat counters the low beam filling argument. Nevertheless, the true continuum brightness temperature is very likely much larger than the CMB temperature of $T_{\mathrm{CMB}}=2.73 \mathrm{~K}$. We can therefore neglect $T_{\mathrm{CMB}}$ in all further calculations. We can also disregard $T_{\mathrm{ex}}$. LVG models of the ammonia molecule (e.g., Walmsley \& Ungerechts 1983) show that, under normal circumstances, the excitation of inversion lines hardly exceeds $T_{\mathrm{ex}} \sim 50 \mathrm{~K}$ (see also Sect4.3). The continuum brightness temperature therefore dominates the radiative transfer. With those assumptions, Eq.2 can be expressed as

$$
\tau=-\ln \left(1-\frac{\left|T_{\mathrm{L}}\right|}{T_{\mathrm{C}}^{\prime}}\right),
$$

with the approximated $\tau$ denoting the 'apparent optical depth'.

In Figs.1, 2, and 3 the absorption profiles of the ATCA and GBT observations are displayed in units of apparent optical depth (using for both the ATCA continuum flux 
described in Sec.3.1). The Gaussian fits to the spectra (Fig.21) result in peak apparent optical depths of $\sim 0.05-0.18$ (Table2) which indicate optically thin lines. The peak apparent optical depths are similar to what Takano et al. (2005) finds for the $\mathrm{NH}_{3}(1,1)$, $(3,3)$, and $(4,4)$ lines. Our fit to the $(2,2)$ line, however, is about 3 times larger than theirs. We should also note that the integrated apparent optical depths are different due to the larger linewidths reported by Takano et al. (2005).

\subsection{Rotational and Kinetic Temperature}

Using the apparent optical depths, it is possible to derive the ratios of the ammonia column densities $N(J, K)$ and excitation temperatures $T_{\mathrm{ex}}$ for the two states of a given inversion doublet with the equation

$$
\frac{N(J, K)}{T_{\mathrm{ex}}}=1.61 \times 10^{14} \times \frac{J(J+1)}{K^{2} \nu} \times \tau \times \Delta v_{1 / 2}
$$

(Hüttemeister et al. 1995b; $\nu$ in GHz; $\Delta v_{1 / 2}$ : full width half maximum [FWHM] linewidth in $\mathrm{km} \mathrm{s}^{-1}$ ). Normalizing these with respect to their statistical weights and assuming that the excitation temperatures $T_{\text {ex }}$ are the same for the six observed transitions, the ratio of column densities and excitation temperatures $N / T_{\text {ex }}$ as a function of energy may be described by a Boltzmann law with a specific rotational temperature $T_{\text {rot,JJ' }}$ (or simply $T_{\mathrm{JJ}^{\prime}}$ ) via

$$
\frac{N\left(J^{\prime}, J^{\prime}\right) / T_{\mathrm{ex}}^{\prime}}{N(J, J) / T_{\mathrm{ex}}}=\frac{g_{\mathrm{op}}\left(J^{\prime}\right)}{g_{\mathrm{op}}(J)} \frac{2 J^{\prime}+1}{2 J+1} \exp \left(\frac{-\Delta E}{T_{\mathrm{JJ}}}\right)
$$

for a pair of inversion lines $\mathrm{NH}_{3}\left(\mathrm{~J}^{\prime}, \mathrm{J}^{\prime}\right)$ and $\mathrm{NH}_{3}(\mathrm{~J}, \mathrm{~J})\left[g_{\text {op }}(J)\right.$ is the statistical weight for a given ammonia species, $g_{\mathrm{op}}=1$ for para-ammonia, $g_{\mathrm{op}}=2$ for ortho-ammonia].

The assumption of equal $T_{\mathrm{ex}} \approx T_{\mathrm{ex}}^{\prime}$ values is justified for the para-ammonia $(1,1)$, $(2,2)$, and $(4,4)$ lines according to the radiative transfer models of Walmsley \& Ungerechts (1983) and Danby et al. (1988). For the ortho-ammonia (3,3) and (6,6) inversion transitions, excitation temperatures may vary within a factor of $\sim 2$ as compared to the lower energy para $-\mathrm{NH}_{3}$ transitions.

In Fig. 4 , we show a Boltzmann plot of the ammonia inversion lines toward Arp 220. The GBT and the ATCA data align reasonably well for the $\mathrm{NH}_{3}(1,1)$ and $(2,2)$ transitions. The $\mathrm{NH}_{3}(3,3)$ datum, however, is significantly lower for the GBT observations. The weighted population of the $\mathrm{NH}_{3}(2,2)$ state is larger than that of the $(1,1)$ state which is not expected but has been confirmed by our two independent measurements. The small $\mathrm{NH}_{3}(1,1)$ column density is even more surprising as both transitions, $\mathrm{NH}_{3}(1,1)$ and $(2,2)$, belong to the same 
para-ammonia species. Both transitions have likely similar excitation temperatures and are optically thin (Table2, see also Sect.4.1). This excludes the possibility that saturation effects are responsible for different strengths of the $(1,1)$ and $(2,2)$ transitions.

An explanation for the weak $\mathrm{NH}_{3}(1,1)$ feature could be that an additional, cool ammonia component in Arp 220 contaminates the absorption spectrum by ammonia $(1,1)$ emission. This cloud needs to be cool to avoid significant population of the higher energy levels; $\mathrm{NH}_{3}(2,2)$ through $(6,6)$ appear to have absorption profiles that are well described by a single rotational temperature and are likely little or not affected by any attenuation from cold gas (see the discussion in the last paragraph of this Section). To be seen in emission, the cold component would have to be in the beam but away from the line of sight toward the continuum. The faint emission channels adjacent to the $(1,1)$ absorption profile of the ATCA data may provide an indication for such a cold component.

In spite of this complication and ignoring the unusual profile of the $(1,1)$ line with respect to all other lines, we can estimate the rotational temperature of the gas. Using the Boltzmann diagram (Fig.4), this is performed by a weighted fit through all $(2,2),(3,3)$, $(4,4),(5,5)$, and $(6,6)$ data points $\left(T_{\text {rot }}=T_{23456}\right.$, with an ortho-to-para ammonia abundance ratio of 1 ). Using the ATCA data, we derive $T_{23456}=124 \pm 19 \mathrm{~K}$ and the best fit is shown in the Boltzmann plot in Fig. 4. Note that the fit describes all data well except for the $(1,1)$ ammonia transition. The good fit to the $(2,2)$ though $(6,6)$ data provides confidence that our interpretation and Gaussian fits to the related spectra are reasonable in terms of peak apparent optical depths and linewidths. However, it also points toward a less reliable $\mathrm{NH}_{3}(3,3)$ GBT flux measurement. The three lowest transitions of the GBT spectrum do not line up at all in the Boltzmann plot. In addition, the $\operatorname{GBT~} \mathrm{NH}_{3}(3,3)$ datum strongly deviates from the best fit of the ATCA $\mathrm{NH}_{3}(2,2)$ through $(6,6)$ data points. We therefore use the superior number and quality of the ATCA spectra throughout the rest of our analysis.

Since the lowest energetic ammonia $(0,0)$ transition belongs to the ortho-ammonia species, cold ammonia formation temperatures would be reflected in an enhanced production of ortho- $\mathrm{NH}_{3}$ over para- $\mathrm{NH}_{3}$. In that case one would observe the $(3,3)$ and $(6,6)$ transition data to be significantly elevated from the rotational temperature fit through all points. A $T_{\text {ex }}$ difference between the ortho and para ammonia inversion lines, however, could compensate or strengthen a deviation from a single-line fit in the Boltzmann diagram. Such a difference can be well fitted by a linear function through $\mathrm{NH}_{3}(2,2)$ and higher, which suggests that the ortho-to-para- $\mathrm{NH}_{3}$ ratio does not deviate substantially from unity. In turn, this implies that the ammonia was formed under warm conditions. Takano, Nakai, \& Kawaguchi (2002) show that ortho-to-para- $\mathrm{NH}_{3}$ ratios near unity require formation temperatures exceeding $\sim 30 \mathrm{~K}$.

For low temperatures, rotational temperatures correspond well to kinetic temperatures. 
At higher temperatures, however, populations of more and more non-metastable levels as well as radiative decay result in a deviation from the approximation Walmsley \& Ungerechts (cf. 1983). In Fig. 5 we show the results on the $T_{\text {kin }}-T_{\text {rot }}$ relation for different pairs of inversion transitions. This calculation is based on an LVG radiative transfer model (described in Ott et al. 2005, using the collision rates of Danby et al. 1988). For the $\mathrm{NH}_{3}(1,1)$ and $(2,2)$ transitions, $T_{\text {kin }} \approx T_{12}$ is valid below $\sim 40 \mathrm{~K}$. Using higher transitions, the approximate equality is valid into progressively higher temperature regimes. For that reason, it is preferable to observe high $\mathrm{NH}_{3}$ transitions if they are bright enough. Nevertheless, even if the $T_{\text {kin }} \approx T_{\text {rot }}$ relation is broken, one can still apply the models displayed in Fig. 5 as they only weakly depend on volume density (for details, see Ott et al. 2005). Here we present logarithmic fits of $T_{\text {kin }}$ to the rotational temperatures $T_{12}$ and $T_{36}$, that are based on the lowest para-ammonia $(1,1)$ and $(2,2)$, and the lowest ortho-ammonia $(3,3)$ and $(6,6)$ transitions, respectively:

$$
T_{\text {kin }}=6.06 \times \exp \left(0.061 T_{12}\right)
$$

and

$$
T_{\text {kin }}= \begin{cases}T_{36} & \text { for } T_{36} \lesssim 50 \mathrm{~K} \\ 28.9 \times \exp \left(0.015 T_{36}\right) & \text { for } T_{36} \gtrsim 50 \mathrm{~K}\end{cases}
$$

Both fits to the LVG solutions are shown in Fig. 5 as dashed lines to their corresponding curves.

For Arp 220, we determine the rotational temperature across five transitions, rather than only a pair. Eq. 6 cannot be applied as the $(1,1)$ level seems to be an outlier in Arp 220 and was excluded from our $T_{\text {rot,23456 }}$ fit. We are using Eq.7 instead. This rotational temperature is calculated over a much larger energy difference of the $(3,3)$ and $(6,6)$ levels, rather than the smaller difference between $(1,1)$ and $(2,2)$. In addition, a Boltzmann fit through $(3,3)$ and $(6,6)$ provides the same $124 \mathrm{~K}$ rotational temperature as the fit through $(2,2)-(6,6)$. For that reason, we expect that the conversion of Eq. 7 is a good approximation for the kinetic temperature of the ammonia-traced gas in Arp 220 and we derive $T_{\text {kin }}=(186 \pm 55) \mathrm{K}$.

Such a kinetic temperature is similar to that found in the more nearby 'normal' starburst galaxy NGC 253, which exhibits $T_{\text {kin }}=130-210 \mathrm{~K}$ (Ott et al. 2005). Other starburst galaxies, such as Maffei 2, and M 82 (Henkel et al. 2000; Weiß et al. 2001; Takano, Nakai, \& Kawaguchi 2002; Mauersberger et al. 2003) show lower rotational temperatures than NGC 253, which must also result in lower kinetic temperatures.

For comparison, Downes \& Eckart (2007) report an intrinsic hot dust temperature com- 
ponent with $T_{\text {dust }}=170 \mathrm{~K}$ toward the western nucleus of Arp 220. This is comparable to what we obtain for $\mathrm{NH}_{3}$, but might not emerge from the same physical feature (see Sect.4.4). Dust temperatures are somewhat lower in the outer molecular ring and over the entire body of Arp 220. ISO observations show two dust temperature components with 47 and $120 \mathrm{~K}$ (Klaas et al. 1997). The gas thus has a kinetic temperature component as high as that of the hottest dust component in Arp 220. Recent SMA (Sakamoto et al. 2008; Aalto et al. 2009), ASTE (Imanishi et al. 2010), and JCMT data (Greve et al. 2009; Papadopoulos et al. 2010a) of highly excited, dense gas show typical temperatures of $\sim 45-120 \mathrm{~K}$ in the densest, $\sim 10^{6} \mathrm{~cm}^{-3}$ components, likely the gas closest to the cores. The temperature of the gas phase we determine is somewhat higher and may arise within the surrounding ring or disk (see Sects.4.3 and 4.4, see also Fig.6). No direct temperature measurement has so far been reported for this physical feature.

Given the rotational temperatures, we can now estimate properties of any ammonia that contributes emission to explain the low absorbed flux of the $(1,1)$ line relative to the $(2,2)$ line. Following the Boltzmann plot (Fig.(4), and assuming that the derived $T_{23456}$ rotational temperature can be extrapolated to a hypothetical $(1,1)$ column density, one would expect a $\sim 4$ times larger column density of the $(1,1)$ line than actually measured in absorption. According to Eq.4, this would result in an integrated apparent optical depth $\sim 4$ times larger. This missing integrated apparent optical depth must be compensated for by emission of order $\sim 20 \mathrm{mJy}$. The gas responsible for such emission would have to be cold to avoid contaminating the other inversion profiles. For an upper limit, let us assume that the $\mathrm{NH}_{3}(2,2)$ line is uncontaminated at a level two times below the one actually measured and that, from that level, the additional emission component raises the (negative) flux to the measured value. The emission line would then require a strength of $\sim 6 \mathrm{mJy}$. Using this value as an upper limit to the $\mathrm{NH}_{3}(2,2)$ emission component, we can derive an upper limit to the temperature from the $(1,1)$ and $(2,2)$ lines. Using the equations for ammonia emission as provided in Ott et al. (2005), we estimate an upper rotational temperature limit of $T_{12} \lesssim 20 \mathrm{~K}$. With Eq.6, this translates to an upper limit of the kinetic temperature of the same order of $T_{\text {kin }} \lesssim 20 \mathrm{~K}$. This scenario can be proven by observations at high spatial resolution $\left(\lesssim 0.5^{\prime \prime}\right)$ that allow direct imaging of emission components offset to the lines of sight toward the bright continuum sources. The EVLA may provide such observations in its extended configurations. Alternatively, $\mathrm{mm} / \mathrm{submm}$ interferometers such as ALMA and PdBI may be able to confirm the existence of this cold component. The ammonia inversion lines, however, do not fall in their wavebands and the predicted cold gas component would need to be revealed via low energy, high frequency transitions of other suitable molecular tracers. 


\subsection{Gas Densities}

The excitation temperatures of ammonia in ULIRGs is still unclear. An indication of a reasonable range might be given by the calculations of Walmsley \& Ungerechts (1983) (see their fig. 3, and using their own collision rates). For the ratio of an $\mathrm{NH}_{3}$ concentration to velocity gradient of $10^{-4} \mathrm{~cm}^{-3}\left(\mathrm{~km} \mathrm{~s}^{-1} \mathrm{pc}^{-1}\right)^{-1}$, they show that the excitation temperatures of the ammonia inversion lines span $T_{\mathrm{ex}} \sim 10-50 \mathrm{~K}$, except for a poorly confined range of parameters where the ammonia $(3,3)$ line exhibits maser emission. All of the inversion states have similar excitation temperatures at a given density. ULIRGs such as Arp 220, however, could show substantially different excitation temperatures due to their high gas densities, high kinetic temperatures, and strong velocity gradients. Nevertheless, assuming that the Walmsley \& Ungerechts (1983) calculations provide at least an indication of the possible range, we can derive ammonia column densities using Eq. 4. The results are listed in Table3, The individual inversion states exhibit column densities of order $\sim 10^{16} \mathrm{~cm}^{-2}$ for $T_{\text {ex }}=50 \mathrm{~K}$ and $\sim 10^{15} \mathrm{~cm}^{-2}$ for $T_{\text {ex }}=10 \mathrm{~K}$, which add up to $(7.8 \pm 0.4) \times 10^{16} \mathrm{~cm}^{-2}$ and $(1.6 \pm 0.1) \times 10^{16} \mathrm{~cm}^{-2}$ for the two excitation temperature values, respectively. The relation scales linearly with $T_{\text {ex }}$, so even a high value of $100 \mathrm{~K}$ or $150 \mathrm{~K}$ would only modestly increase the column densities. The $(0,0)$ ortho-ammonia level is degenerate and does not exhibit an inversion line. However, it is the lowest energy state of ammonia and thus has a significant population. To estimate the amount of ammonia in the $(0,0)$ state, we extrapolate the $T_{23456}=(124 \pm 19) \mathrm{K}$ rotational temperature in the Boltzmann diagram (Fig. 4, assuming an ortho-to-para- $\mathrm{NH}_{3}$ ratio of unity) and derive $(0,0)$ columns of $(6.0 \pm 0.9) \times 10^{15} \mathrm{~cm}^{-2}$ and $(1.2 \pm 0.2) \times 10^{15} \mathrm{~cm}^{-2}$ for excitation temperatures of $50 \mathrm{~K}$ and $10 \mathrm{~K}$, respectively. Adding this to the $(1,1)$ through $(6,6)$ columns, we derive total ammonia columns of $(8.4 \pm 0.5) \times 10^{16} \mathrm{~cm}^{-2}$ and $(1.7 \pm 0.1) \times 10^{16} \mathrm{~cm}^{-2}$ for values of $T_{\mathrm{ex}}=50 \mathrm{~K}$ and $10 \mathrm{~K}$, respectively (see Table 3 ). This value neglects all higher level populations, a valid assumption given the negligible contributions that these will make to the entire column if the rotational temperature stays the same for $J>6$. For example, the $(7,7)$ inversion line is $538 \mathrm{~K}$ above the ground state; by extrapolating $T_{\text {rot }}$ via Eq. 5 in the Boltzmann diagram (Fig.4), we see that the $\mathrm{NH}_{3}(7,7)$ line would fall at a $N / T_{\text {ex }}$ that is $\sim 14$ times below the $\mathrm{NH}_{3}(6,6)$ value.

As shown in Fig. 3 and in Table2, some peak and integrated apparent optical depths differ between the ATCA and GBT observations. In the following, we try to derive how the properties of the gas have changed if the different spectra are due to a time variation of the gas in front of the line of sight to the continuum (the GBT data were observed about a year after the ATCA data). To start, we use Eq.4 to determine $N / T_{\text {ex }}$, which is directly proportional to the integrated apparent optical depths. According to Table2, this translates to ATCA over GBT column density ratios of $\sim 1.3$ and 2.6 for the $\mathrm{NH}_{3}(2,2)$ and $(3,3)$ transitions, respectively, at a given $T_{\text {ex }}$. The total ammonia columns depend on 
the temperature assumed, but, as shown in Fig.4, the GBT data points are not reliable enough to determine a temperature as the $\mathrm{NH}_{3}(1,1)$ line has very different properties. If the $(2,2)$ and $(3,3)$ lines are representative of the gas phase measured by the GBT, however, we can estimate a rotational temperature from the slope and we derive a cold $T_{23} \sim 42 \mathrm{~K}$. Extrapolating this to higher transitions via Eq. 5 and adding the $\mathrm{NH}_{3}(1,1)$ will then provide $\sim 28 \times 10^{15} \mathrm{~cm}^{-2}$ for $T_{\mathrm{ex}}=50$ and $\sim 6 \times 10^{15} \mathrm{~cm}^{-2}$ for $T_{\mathrm{ex}}=10$, or $\sim 2.5$ times less than what we estimate from the ATCA data (see Table 3 ). This is the variation of the ammonia column that potentially may have modified the absorption profile between the ATCA and GBT measurements over the time of a year.

Eventually, we can use the total column densities to derive ammonia volume densities. Downes \& Solomon (1998) show that the bulk of the molecular gas in Arp 220 is found in a ring or disk (the light gray shaded area in Fig.6). In their model, the size of this ring or disk is about $\sim 700 \mathrm{pc}$ when measured from the western edge closest to the observer toward the eastern nucleus, the farthest continuum source against which we see the $\mathrm{NH}_{3}$ absorption. This geometry leads to $\mathrm{NH}_{3}$ volume densities of $f_{\mathrm{V}}^{-0.5} \times(3.9 \pm 0.2) \times 10^{-5} \mathrm{~cm}^{-3}$ and $f_{\mathrm{V}}^{-0.5} \times(0.8 \pm 0.1) \times 10^{-5} \mathrm{~cm}^{-3}$ for the $T_{\mathrm{ex}}=50 \mathrm{~K}$ and $10 \mathrm{~K}$ limits, respectively $\left(f_{\mathrm{V}}\right.$ is the volume filling factor of the dense gas). If the line of sight through the molecular gas is substantially smaller, i.e. if the absorption occurs mainly against the western core of Arp 220 (see Sect.4.4), the volume densities increase by a factor of $\sim 3$.

We can now estimate the volume densities of molecular hydrogen that is traced by the ammonia absorption lines. To do so, we have to assume a fractional abundance of ammonia. Ott et al. (2005) and Mauersberger et al. (2003) show that for a sample of nearby, nonULIRG starburst galaxies, $\left[N\left(\mathrm{NH}_{3}\right) / N\left(\mathrm{H}_{2}\right)\right]$ hovers around $10^{-8}$. If this value is applicable to the dense gas in Arp 220, the $\mathrm{H}_{2}$ densities are of order $f_{\mathrm{V}}^{-0.5} \times(1-4) \times 10^{3} \mathrm{~cm}^{-3}$. For a filling factor of unity, this is similar to the environment of the Sgr B2 region near the Galactic Center (Hüttemeister et al. 1995b) and close to the critical density of ammonia which is $\sim 2 \times 10^{3} \mathrm{~cm}^{-3}$ (Rohlfs \& Wilson 2004). Scoville, Yun, \& Bryant (1997), however, estimate a density of $\sim 2 \times 10^{4} \mathrm{~cm}^{-3}$ for the molecular ring in Arp 220. If the ammonia absorption lines trace the same gas, the volume filling factor would be of order $f_{\mathrm{V}} \sim 0.01$.

In Sect.4.2, we speculate that the small absorption depth of the $(1,1)$ line relative to the other $\mathrm{NH}_{3}$ lines might be caused by an additional, cold layer of ammonia in Arp 220. The component would have to be located inside the beam but should be displaced from the lines of sight toward the continuum sources and thus be seen in emission. If this line has a width of $\sim 200 \mathrm{~km} \mathrm{~s}^{-1}$, such as to compensate some of the absorption line, the assumed strength can be converted to a column density in the $(1,1)$ line of $\sim 1 \times 10^{14} \mathrm{~cm}^{-2}$. With $T_{12}=20 \mathrm{~K}$ (Sect.4.2) and using the Equation (A15) in Ungerechts, Winnewisser, \& Walmsley (1986), 
this can be extrapolated to a total column of $N_{\text {emission }} \sim 2 \times 10^{14} \mathrm{~cm}^{-2}$. The hypothetical emission component thus has a column density more than 2 orders of magnitude smaller than the ammonia layer that is seen in absorption against the background continuum.

\subsection{Gas Kinematics}

The ammonia linewidths, $\sim 230-430 \mathrm{~km} \mathrm{~s}^{-1}\left(\mathrm{NH}_{3}(1,1): 121 \mathrm{~km} \mathrm{~s}^{-1}\right)$, are at the upper end and even wider than those found in nearby 'normal' starburst galaxies, such as NGC $253\left(\sim 250 \mathrm{~km} \mathrm{~s}^{-1}\right.$; Ott et al. 2005), Maffei $2\left(\sim 150 \mathrm{~km} \mathrm{~s}^{-1}\right)$, or IC $342\left(\sim 50 \mathrm{~km} \mathrm{~s}^{-1}\right.$; Mauersberger et al. 2003) when averaged over the nuclear regions. Toward Arp 220, molecular emission lines such as $\mathrm{CO}$ and $\mathrm{HCO}^{+}$and $\mathrm{HNC}$ (e.g., see the summary in Greve et al. 2009) tend to be broadened to $\sim 320-430 \mathrm{~km} \mathrm{~s}^{-1}$, as are highly excited CO and CS transitions (Sakamoto et al. 2008; Greve et al. 2009; Papadopoulos et al. 2010a, e.g.) which show linewidths of $\sim 300 \mathrm{~km} \mathrm{~s}^{-1}$ for each of the nuclear components. Imaging of the CO $(J=2 \rightarrow 1)$ distribution with sub-arcsecond resolution (Scoville. Yun. \& Bryant 1997; Downes \& Solomon 1998) provides information on the dynamical properties of the molecular gas. Toward the east of the active region that encompasses the two galactic nuclei, the velocity range is $5500-5800 \mathrm{~km} \mathrm{~s}^{-1}$ and toward the west it is $5200-5500 \mathrm{~km} \mathrm{~s}^{-1}$ (Scoville, Yun, \& Bryant 1997). One possibility is that the molecular gas we observe in ammonia, $\sim 5450 \mathrm{~km} \mathrm{~s}^{-1}$ with a linewidth of $\sim 230-430 \mathrm{~km} \mathrm{~s}^{-1}$, has components toward both nuclei. This, however, is unlikely since the absorption spectra are well defined by a one-component Gaussian that cannot be explained by two disks, separated in velocity space well above our velocity resolution. Alternatively, the width may reflect the difference in systemic velocities of the cores, which is $\sim 300 \mathrm{~km} \mathrm{~s}^{-1}$ (e.g. Downes \& Solomon 1998). It is a coincidence that the linewidths of the gas around the individual cores and the velocity difference between the cores is of the same order. This makes it difficult to determine the origin of the gas based on kinematic information alone. The $\mathrm{NH}_{3}$ spectra show similarities to previously measured $\mathrm{OH}$ lines. The absorption lines of rotationally excited $\mathrm{OH}$ exhibit linewidths of $\sim 250-370 \mathrm{~km} \mathrm{~s}^{-1}$ (Henkel et al. 1986, 1987; Salter et al. 2008) which is like the linewidths obtained from $\mathrm{NH}_{3}$ and, as $\mathrm{NH}_{3}, \mathrm{OH}$ absorption has no clear velocity imprint of either nucleus. Both ammonia and $\mathrm{OH}$ may therefore trace the same molecular material either from a single nucleus, such as the linewidth suggests, or, alternatively, in the common disk in which the two nuclei are embedded, as suggested by the systemic velocities.

The gas in the dense cores has volume densities of $\sim 10^{6} \mathrm{~cm}^{-3}$ (see Sect.4.3), but we estimate a 2-3 orders of magnitude lower density for the $\mathrm{NH}_{3}$ traced gas (Table 3). A combination of the systemic line velocities, the derived densities, and the temperature argues 
that the ammonia- (and $\mathrm{OH}-$ ) traced gas is more likely to be stored in the inner ring or disk that surrounds both nuclei (see Fig.66). Unfortunately, as for ammonia, only single-dish data of rotationally excited $\mathrm{OH}$ have been published to date and its distribution with respect to the nuclei in Arp 220 has not yet been directly imaged. Sub-arcsecond mapping of the components with the EVLA or ALMA is needed to at least determine the absorption profiles against each nucleus. Such observations will be crucial to obtain a better model of the layered gas distribution in Arp 220.

\section{Summary}

In this article, we have presented ATCA and GBT observations of ammonia toward the ultraluminous far-infrared galaxy Arp 220. We conclude the following:

1. We detect all observed ammonia lines $(J, K)=(1,1),(2,2),(3,3),(4,4),(5,5)$, and $(6,6)$, toward the bright continuum in absorption.

2. The detected ammonia linewidths are in the range $\sim 120-430 \mathrm{~km} \mathrm{~s}^{-1}$, in agreement with those of other molecules. We cannot confirm the extremely wide lines reported by Takano et al. (2005).

3. Based on the ATCA ammonia data (but excluding the $\mathrm{NH}_{3}(1,1)$ line), we derive the rotational temperature of the molecular gas to be $(124 \pm 19) \mathrm{K}$. This translates to a kinetic temperature of $T_{\text {kin }}=(186 \pm 55) \mathrm{K}$. The kinetic temperature of $\mathrm{NH}_{3}$ is similar to that found in the nucleated starburst galaxy NGC 253.

4. We see no evidence for an ortho-to-para-ammonia ratio other than unity. This implies ammonia formation temperatures exceeding $\sim 30 \mathrm{~K}$.

5. Combined column densities of the measured lines plus the extrapolated $(0,0)$ column add up to $(1.7 \pm 0.1) \times 10^{16} \mathrm{~cm}^{-2}$ and $(8.4 \pm 0.5) \times 10^{16} \mathrm{~cm}^{-2}$ for assumed excitation temperatures of $10 \mathrm{~K}$ and $50 \mathrm{~K}$, respectively. We estimate the volume density of the molecular gas that is traced by ammonia to be $\sim f_{\mathrm{V}}^{-0.5} \times(1-4) \times 10^{3} \mathrm{~cm}^{-3}\left(f_{\mathrm{V}}\right.$ : volume filling factor). Together with the temperature estimate and the fact that the spectra are well described by single-component Gaussians, we conclude that the bulk of this moderately dense gas likely surrounds both starburst nuclei.

6. Widespread gas, based on low excitation $\mathrm{CO}$, exhibits densities that are about two orders of magnitude less than the ammonia-traced gas layer. This component defines the outer disk of Arp 220. In contrast, the gas close to the two starburst nuclei is 
characterized by densities two orders of magnitude larger than the ammonia-traced gas. The gas that we see via ammonia is thus likely a layer that is located between the nuclei and the outer envelope. The geometry is most likely a ring or disk that surrounds both nuclei. The temperature profile appears to have a local maximum at the position of the gas that is traced by ammonia.

7. The $\mathrm{NH}_{3}(1,1)$ line is weaker than expected for thermal gas based on all other measured $\mathrm{NH}_{3}$ transitions. A possible explanation for this unusual behavior could be an additional, cold, $\lesssim 20 \mathrm{~K}$ component of molecular gas, located inside the beam but displaced from the lines of sight toward the continuum sources in Arp 220. This gas, if present, would have to be at an estimated column density of $N_{\text {emission }} \sim 2 \times 10^{14} \mathrm{~cm}^{-2}$ to sufficiently mask the $(1,1)$ absorption. The cool temperature is required to keep the masking of the other lines to a minimum.

8. We also detect an absorption feature adjacent in frequency to the $\mathrm{NH}_{3}(3,3)$ line in the ATCA data but not in the GBT data. This absorption feature may be interpreted as an $\mathrm{OH}^{2} \Pi_{3 / 2} J=9 / 2 F=4-4$ transition, or the combination of the $\mathrm{F}=4-4$ and $\mathrm{F}=5-5$ $\mathrm{OH}$ doublet. Both options yield line parameters similar to that of ammonia, including a rotational temperature that is about 2 times larger for $\mathrm{OH}$. If this detection holds, it would be the first extragalactic evidence for this line which has so far only been seen toward Galactic compact H II regions such as W3(OH).

9. The ATCA and GBT data show some differences in their line shapes. This is most likely an instrumental effect of the two very different telescopes and observing procedures. We cannot exclude, however, that there is intrinsic time variability of the gas distribution in Arp 220 along the narrow lines of sight toward the absorbing background structure. If this is the case, then the GBT data suggests gas with a $\sim 3$ times cooler rotational temperature than that of the ATCA measurement, and the column density would be $\sim 2.5$ times lower at the time when the GBT observations were taken (a year after the ATCA observations). Given the typical cloud and continuum size scales of a few to tens of parsec and the velocities of a few hundred $\mathrm{km} \mathrm{s}^{-1}$, however, drastic variations of the absorption spectra on annual timescales seem to be unlikely and an instrumental effect is more plausible.

We thank Fabian Walter for his comments on the manuscript. C. H. thanks ATNF for support during his time spent at ATNF and ATCA. This research has made use of the NASA/IPAC Extragalactic Database (NED), which is maintained by the Jet Propulsion Laboratory, Caltech, under contract with the National Aeronautics and Space Administration (NASA) and NASA's Astrophysical Data System Abstract Service (ADS). 
Facilities: ATCA,GBT.

\section{REFERENCES}

Aalto, S., Wilner, D., Spaans, M., Wiedner, M. C., Sakamoto, K., Black, J. H., \& Caldas, M. 2009, A\&A, 493, 481

Araya, E., Baan, W. A., \& Hofner, P. 2004, ApJS, 154, 541

Baan, W. A., Wood, P. A. D., \& Haschick, A. D. 1982, ApJ, 260, L49

Baudry, A., \& Menten, K. M. 1995, A\&A, 298, 905

Bridge, C. R., et al. 2007, ApJ, 659, 931

Canalizo, G., \& Stockton, A. 2001, ApJ, 555, 719

Cole, S., et al. 2001, MNRAS, 326, 255

Danby, G., Flower, D. R., Valiron, P., Schilke, P., \& Walmsley, C. M. 1988, MNRAS, 235, 229

Darling, J., Giovanelli, R. 2002, ApJ, 572, 810

Dasyra, K. M., et al. 2007, ApJ, 657, 102

Downes, D., \& Eckart, A. 2007, A\&A, 468, L57

Downes, D., \& Solomon, P. M. 1998, ApJ, 507, 615

Flores, H., Hammer, F., Elbaz, D., Cesarsky, C. J., Liang, Y. C., Fadda, D., \& Gruel, N. 2004, A\&A, 415, 885

Gao, Y. \& Solomon, P. M. 2004a, ApJ, 606, 271

Gao, Y. \& Solomon, P. M. 2004b, ApJS, 152, 63

Graciá-Carpio, J., García-Burillo, S., Planesas, P., \& Colina, L. 2006, ApJ, 640, L135

Gerin, M., \& Phillips, T. G. 1998, ApJ, 509, L17

Greve, T. R., Papadopoulos, P. P., Gao, Y., \& Radford, S. J. E. 2009, ApJ, 692, 1432

Henkel, C., Guesten, R., \& Batrla, W. 1986, A\&A, 168, L13 
Henkel, C., Guesten, R., \& Baan, W. A. 1987, A\&A, 185, 14

Henkel, C., Mauersberger, R., Peck, A. B., Falcke, H., \& Hagiwara, Y. 2000, A\&A, 361, L45

Hopkins, P. F., Younger, J. D., Hayward, C. C., Narayanan, D., \& Hernquist, L. 2010, MNRAS, 402, 1693

Hutchings, J. B., \& Neff, S. G. 1988, AJ, 96, 1575

Hüttemeister, S., Henkel, C., Mauersberger, R., Brouillet, N., Wiklind, T., \& Millar, T. J. 1995, A\&A, 295, 571

Hüttemeister, S., Wilson, T. L., Mauersberger, R., Lemme, C., Dahmen, G., \& Henkel, C. 1995, A\&A, 294, 667

Imanishi, M., Nakanishi, K., Yamada, M., Tamura, Y., \& Kohno, K. 2010, PASJ, 62, 201

Klaas, U., Haas, M., Heinrichsen, I., \& Schulz, B. 1997, A\&A, 325, L21

Lonsdale, C. J., Diamond, P. J., Thrall, H., Smith, H. E., \& Lonsdale, C. J. 2006, ApJ, 647, 185

Madau, P., Pozzetti, L., \& Dickinson, M. 1998, ApJ, 498, 106

Martín, S., et al. 2011, A\&A, 527, A36

Matsushita, S., et al. 2009, ApJ, 693, 56

Mauersberger, R., Henkel, C., Weiß, A., Peck, A. B., \& Hagiwara, Y. 2003, A\&A, 403, 561

Mundell, C. G., Ferruit, P., \& Pedlar, A. 2001, ApJ, 560, 168

Nagar, N. M., Wilson, A. S., Falcke, H., Veilleux, S., \& Maiolino, R. 2003, A\&A, 409, 115

Norris, R. P. 1988, MNRAS, 230, 345

Ott, J., Weiß, A., Henkel, C., \& Walter, F. 2005, ApJ, 629, 767

Papadopoulos, P. P., \& Seaquist, E. R. 1998, ApJ, 492, 521

Papadopoulos, P. P., Isaak, K., \& van der Werf, P. 2010, ApJ, 711, 757

Parra, R., Conway, J. E., Diamond, P. J., Thrall, H., Lonsdale, C. J., Lonsdale, C. J., \& Smith, H. E. 2007, ApJ, 659, 314 
Rohlfs, K., \& Wilson, T. L. 2004, Tools of Radio Astronomy, 4th revised and enlarged edition, Springer Verlag, Berlin

Rovilos, E., Diamond, P. J., Lonsdale, C. J., Lonsdale, C. J., \& Smith, H. E. 2003, MNRAS, 342,373

Sakamoto, K., Scoville, N. Z., Yun, M. S., Crosas, M., Genzel, R., \& Tacconi, L. J. 1999, ApJ, 514, 68

Sakamoto, K., et al. 2008, ApJ, 684, 957

Sakamoto, K., et al. 2009, ApJ, 700, L104

Salter, C. J., Ghosh, T., Catinella, B., Lebron, M., Lerner, M. S., Minchin, R., \& Momjian, E. 2008, AJ, 136, 389

Sanders, D. B., \& Mirabel, I. F. 1996, ARA\&A, 34, 749

Schilke, P., Walmsley, C. M., Pineau Des Forets, G., Roueff, E., Flower, D. R., \& Guilloteau, S. 1992, A\&A, 256, 595

Scoville, N. Z., Yun, M. S., \& Bryant, P. M. 1997, ApJ, 484, 702

Smith, H. E., Lonsdale, C. J., Lonsdale, C. J., \& Diamond, P. J. 1998, ApJ, 493, L17

Solomon, P. M., Radford, S. J. E., \& Downes, D. 1990, ApJ, 348, L53

Solomon, P. M., Downes, D., \& Radford, S. J. E. 1992, ApJ, 387, L55

Solomon P. M., Downes D., Radford S. J. E., Barrett J. W. 1997, ApJ, 478, 144

Takano, S., Nakai, N., \& Kawaguchi, K. 2002, PASJ, 54, 195

Takano, S., Nakanishi, K., Nakai, N., \& Takano, T. 2005, PASJ, 57, L29

Ungerechts, H., Winnewisser, G., \& Walmsley, C. M. 1986, A\&A, 157, 207

Veilleux, S., et al. 2009, ApJS, 182, 628

Walmsley, C. M. \& Ungerechts, H. 1983, A\&A, 122, 164

Wampfler, S. F., et al. 2010, A\&A, 521, L36

Weiß, A., Neininger, N., Henkel, C., Stutzki, J., \& Klein, U. 2001a, ApJ, 554, L143

Wilson, T. L., Johnston, K. J., \& Mauersberger, R. 1991, A\&A, 251, 220 
Winnberg, A., Walmsley, C. M., \& Churchwell, E. 1978, A\&A, 66, 431

Yao, L., Seaquist, E. R., Kuno, N., \& Dunne, L. 2003, ApJ, 588, 771 


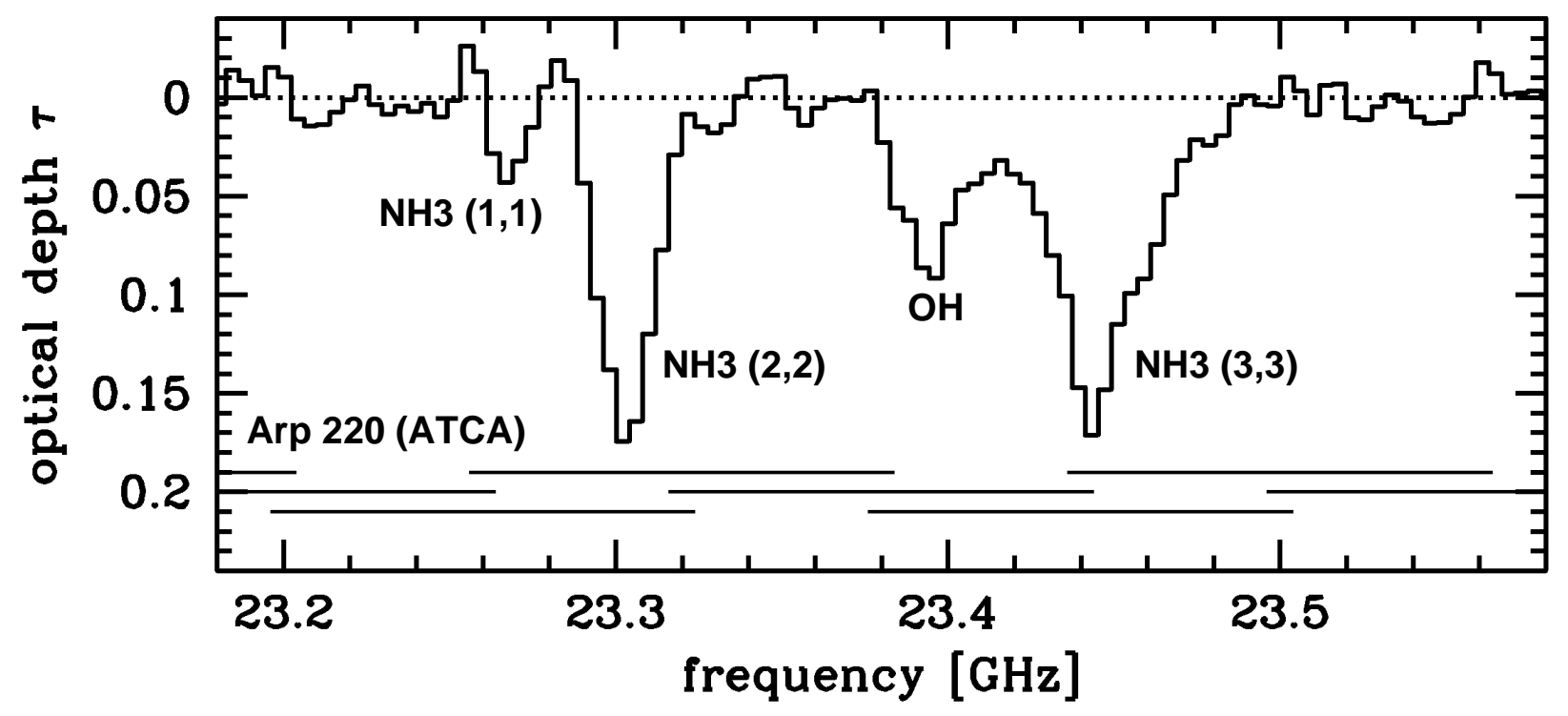

Fig. 1.- ATCA broadband spectrum in the 23.2 to $23.6 \mathrm{GHz}$ range. Units are in apparent optical depth $\tau$. The bars at the bottom indicate the bandwidths and tunings of the different spectral settings. The rms noise of the observations is $\tau_{\mathrm{rms}} \approx 0.013$. 


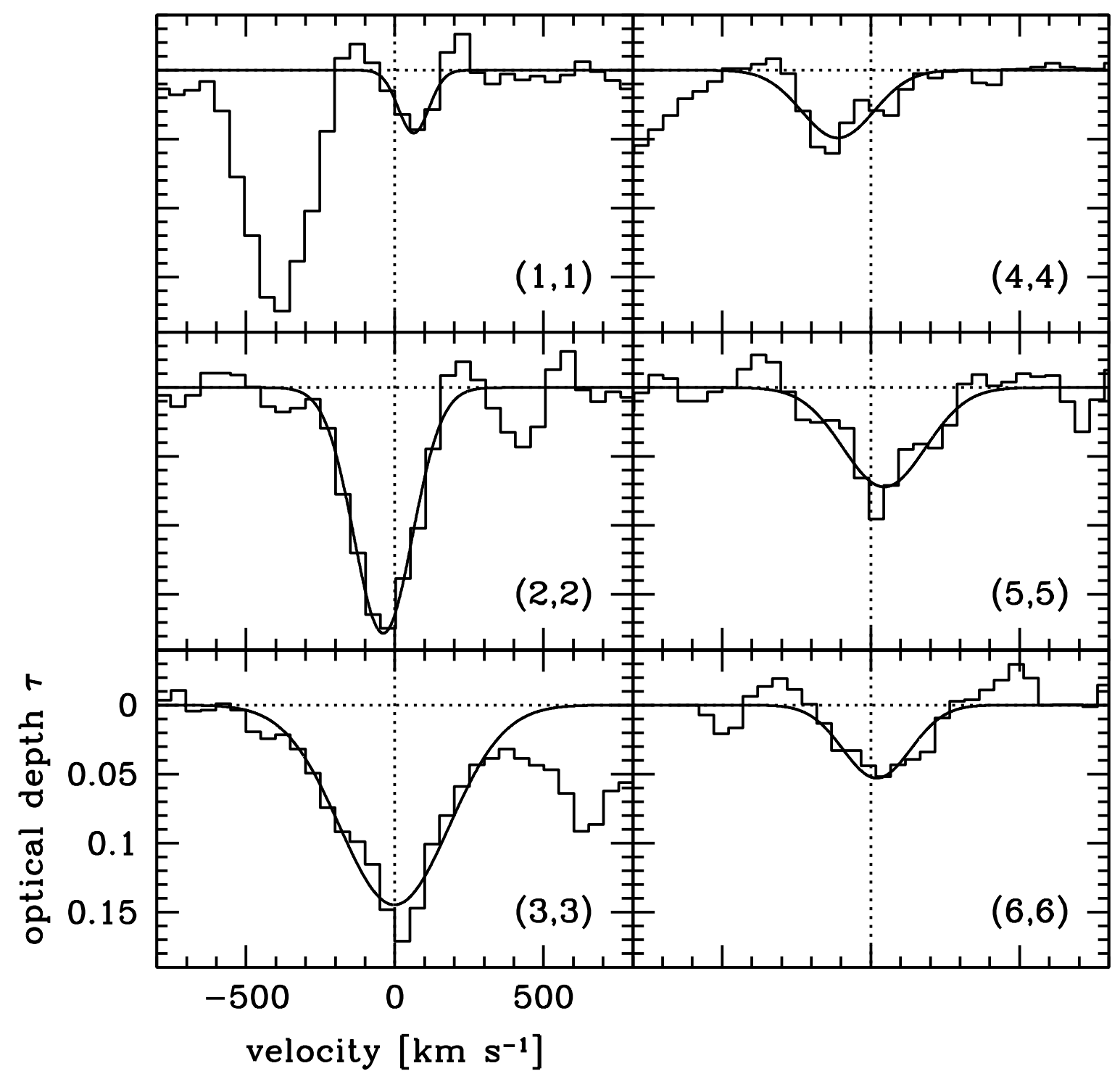

Fig. 2.- Continuum subtracted ammonia absorption ATCA spectra of Arp 220 in units of apparent optical depth $\tau$. Gaussian fits to the lines are overlaid. All panels are on the same scale. 


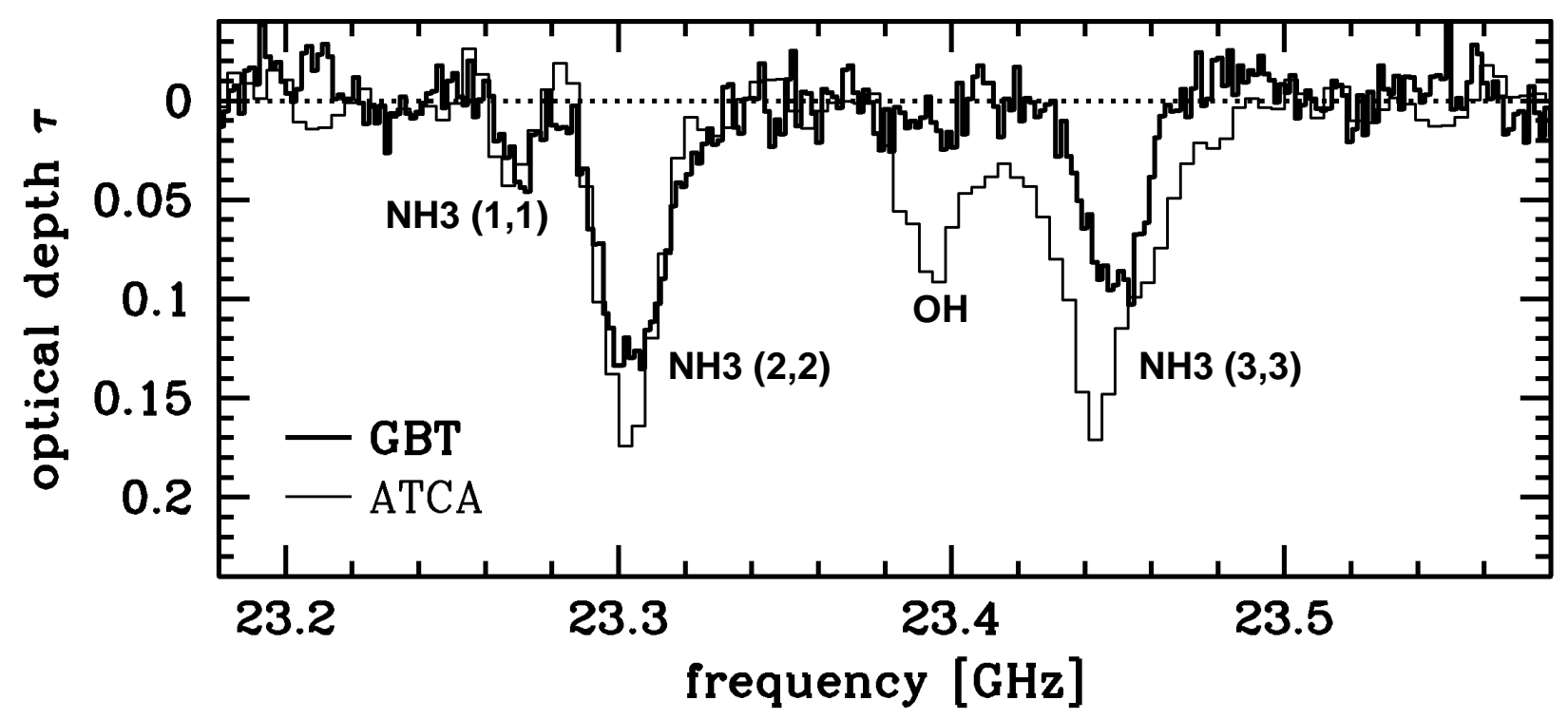

Fig. 3.- GBT broadband spectrum (thick lines) overlaid over the ATCA spectrum. 


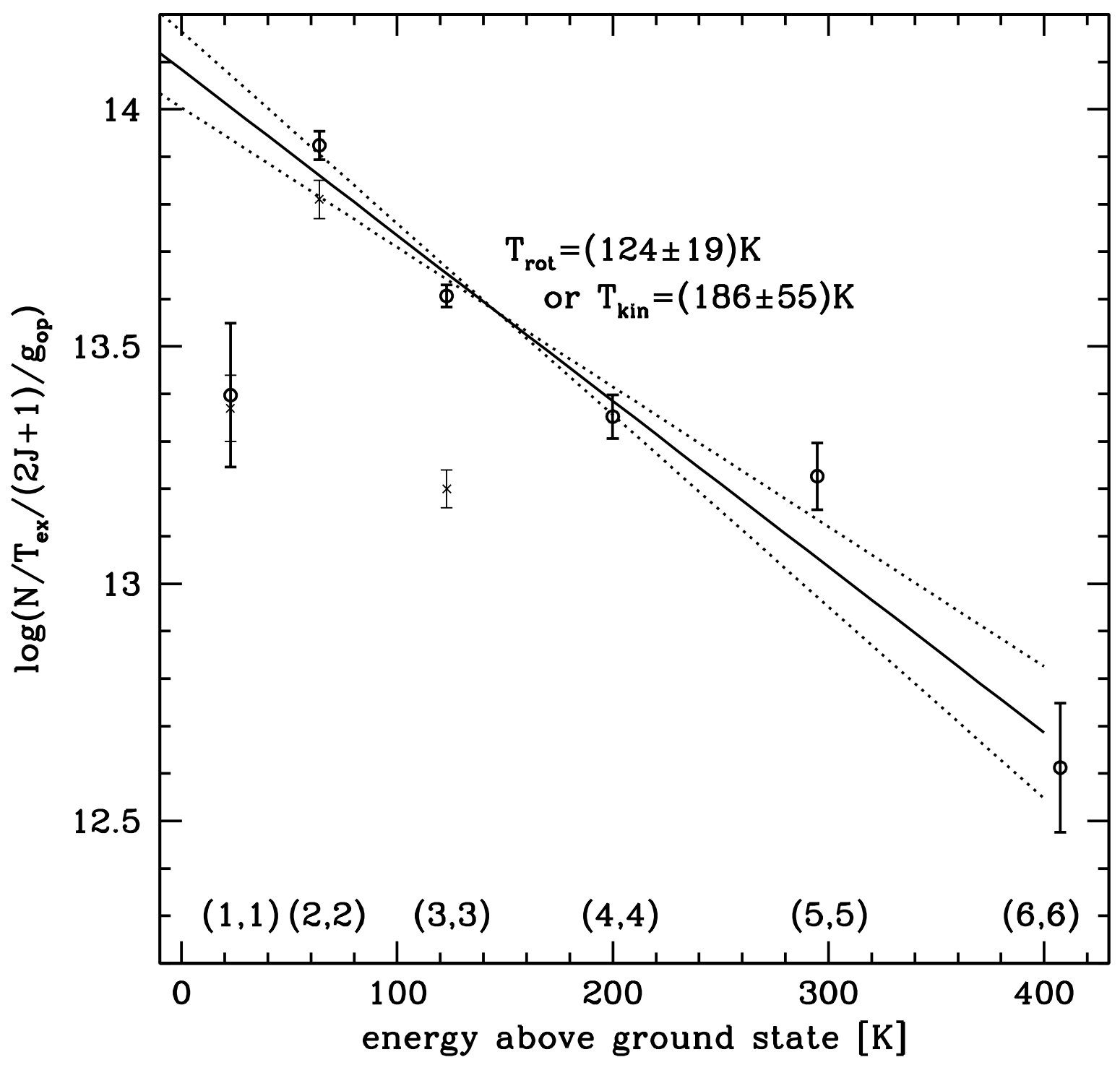

Fig. 4.- Boltzmann plot of the ammonia measurements. The ordinate exhibits the weighted logarithm of $N / T_{\mathrm{ex}}$. We plot the rotational temperature fitted to all but the $(1,1)$ datum with the errors in slope. ATCA data points are marked by bold circles, whereas the GBT data is plotted with thin crosses and errorbars. 


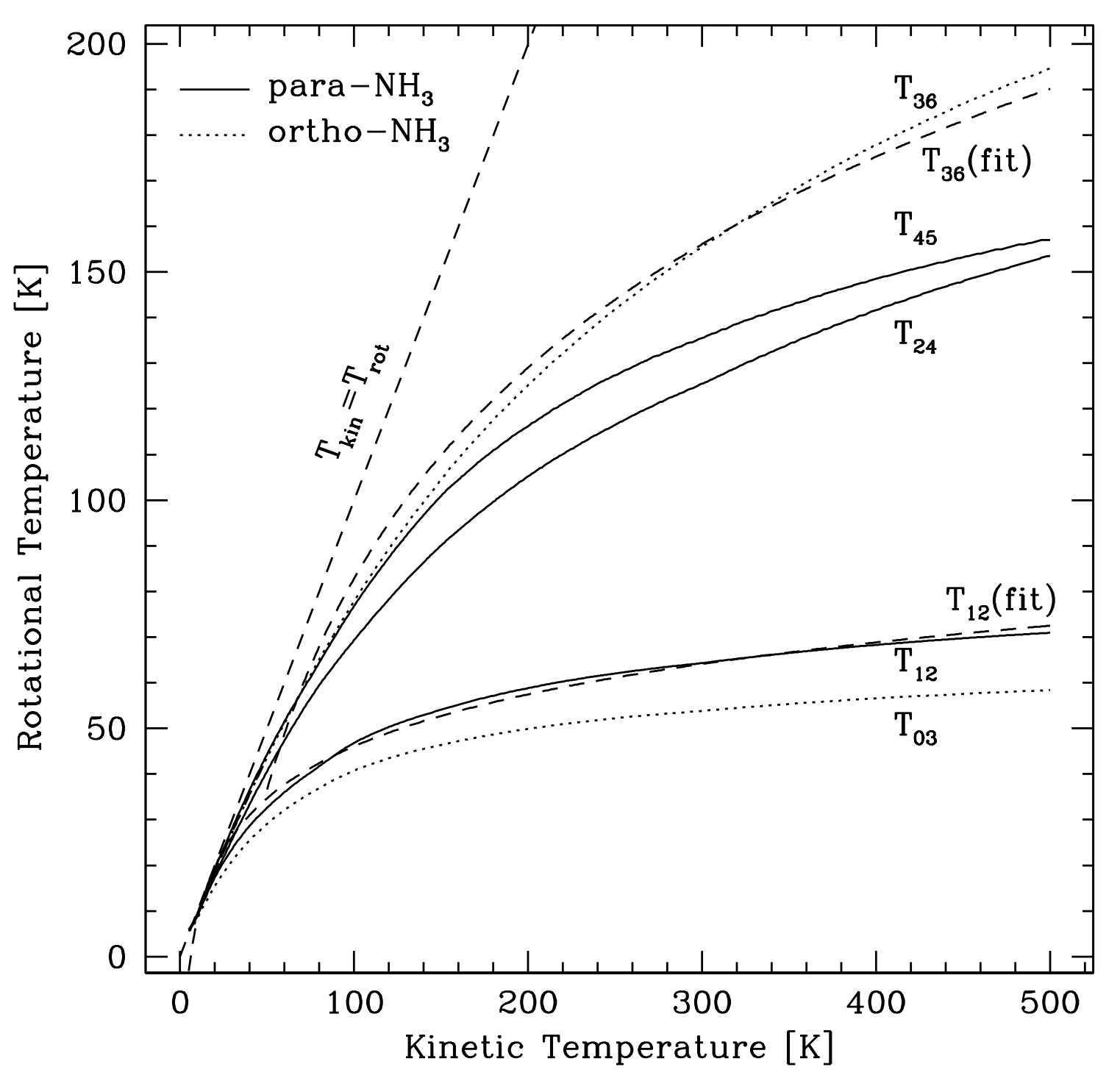

Fig. 5.- LVG results on the conversion between rotational and kinetic ammonia temperatures (solid curves: para-ammonia, dotted curves: ortho-ammonia). The subscripts denote the transitions used for the $T_{\text {rot }}$ determination. The dashed straight line indicates where both temperatures would be identical; $T_{\text {rot }}$ is always underestimating $T_{\text {kin }}$. For $T_{12}$ and $T_{36}$ we show logarithmic fits to the curves in dashed lines. These fits are described in eqs.6, and 7. respectively. 


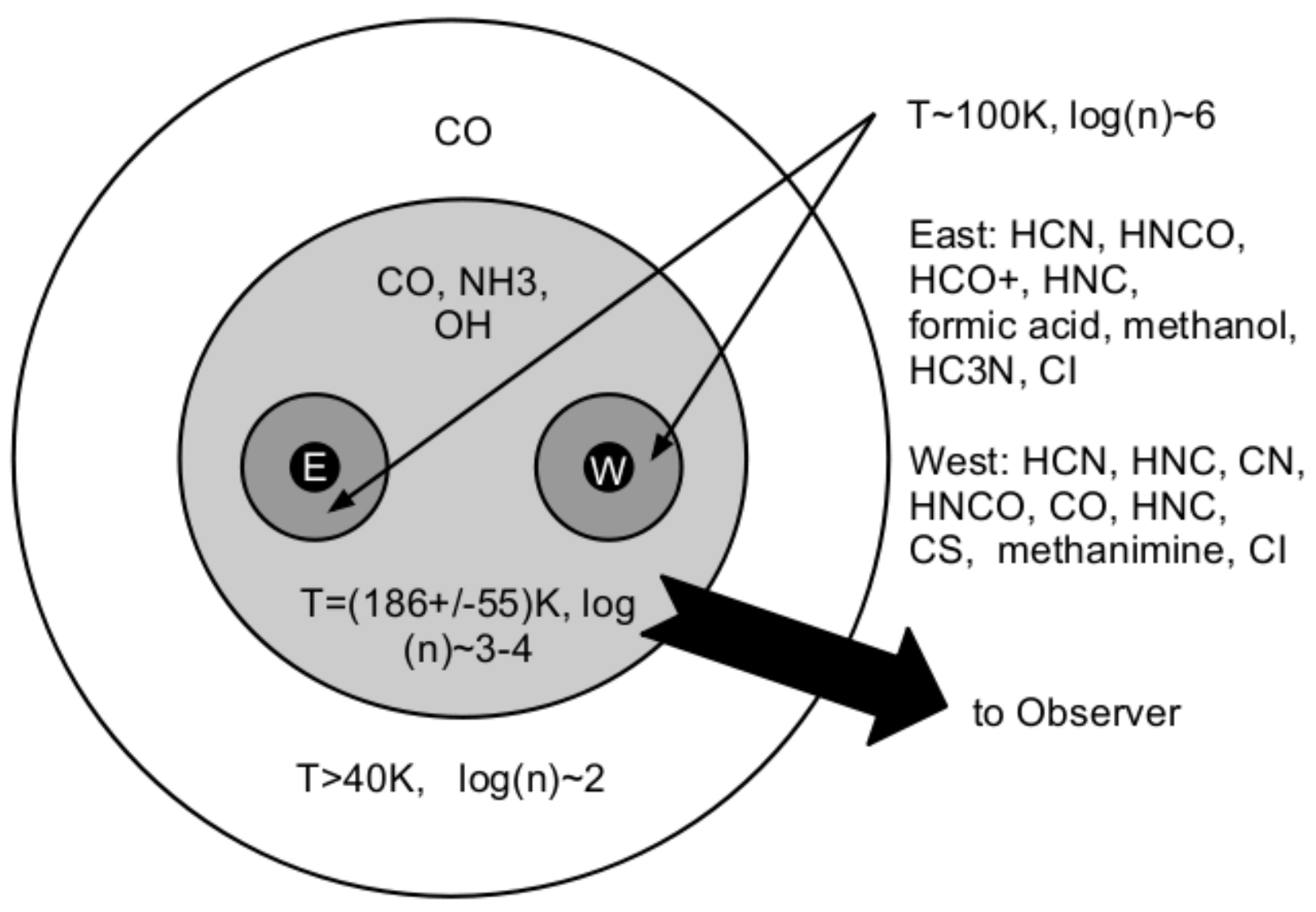

Fig. 6. - A cartoon image of Arp 220. Shown are the different gas phases and some characteristic molecular or atomic tracers. The outer envelope has a lower limit to the temperature and the $\mathrm{NH}_{3}$ component likely resides between the outer envelope and the inner dense regions that surround the eastern and western cores. 
Table 1. Observational Parameters. All observations were directed toward $\mathrm{RA}(\mathrm{J} 2000)=15^{h} 34^{m} 58^{\mathrm{s}} \cdot 10, \operatorname{DEC}(\mathrm{J} 2000)=23^{\circ} 30^{\prime} 11^{\prime \prime} .0$. For the assumed $75 \mathrm{Mpc}$ distance of Arp 220, $1^{\prime \prime}$ corresponds to $0.36 \mathrm{kpc}$.

\begin{tabular}{lllllll}
\hline \hline Telescope & \multicolumn{1}{c}{ Date } & \multicolumn{1}{c}{$\begin{array}{c}\text { Center Frequencies } \\
{[\mathrm{GHz}]}\end{array}$} & $\begin{array}{c}\mathrm{BW} \\
{[\mathrm{MHz}]}\end{array}$ & $\begin{array}{c}\Delta \nu^{\mathrm{b}} \\
{[\mathrm{MHz}]}\end{array}$ & $\begin{array}{c}\text { Beam size } \\
{\left[{ }^{\prime \prime}\right]}\end{array}$ & $\mathrm{NH}_{3}$ Transition \\
\hline ATCA & 2005 Jul 28-31, Aug 01 & $\begin{array}{l}23.14,23.20,23.26,23.32 \\
23.38,23.44,23.50,23.56\end{array}$ & 128 & 4.4 & $31.1 \times 21.1$ & $(1,1),(2,2),(3,3)$ \\
& & $23.665,23.737$ & 128 & 4.4 & $31.2 \times 21.4$ & $(4,4)$ \\
ATCA & 2005 Jul 30 & $24.052,24.124$ & 128 & 4.4 & $30.9 \times 19.8$ & $(5,5)$ \\
ATCA & 2005 Jul 31 & 24.603 & 128 & 4.4 & $10.7 \times 7.7$ & $(6,6)$ \\
ATCA & 2005 Mar 17 and 20 & 800 & 1.56 & 33 & $(1,1),(2,2),(3,3)$ \\
GBT & 2006 Jan 21 and Feb 02 & 23.445 & & & &
\end{tabular}

${ }^{a} \mathrm{BW}$ : bandwidth of each frequency setting

${ }^{\mathrm{b}} \Delta \nu$ : spectral resolution 
Table 2. Ammonia $(1,1)$ through $(6,6)$ line parameters. Velocities are given with respect to the redshift of Arp 220 of $z=0.018126$, or a velocity of $5434 \mathrm{~km} \mathrm{~s}^{-1}$. The parameters of the $\mathrm{OH}^{2} \Pi_{3 / 2} J=9 / 2 F=4-4$ line candidate are also provided.

\begin{tabular}{lcccc}
\hline \hline $\mathrm{NH}_{3}$ line & $\tau^{\text {peak }}$ & $\begin{array}{c}v_{\text {opt,bary }}^{\text {peak }} \\
{\left[\mathrm{km} \mathrm{s}^{-1}\right]}\end{array}$ & $\begin{array}{c}\Delta v_{1 / 2} \\
{\left[\mathrm{~km} \mathrm{~s}^{-1}\right]}\end{array}$ & $\begin{array}{c}\int \tau \mathrm{d} v . \\
{\left[\mathrm{km} \mathrm{s}^{-1}\right]}\end{array}$ \\
\hline \multicolumn{5}{c}{ ATCA } \\
\hline$(1,1)$ & $0.046 \pm 0.012$ & $63 \pm 16$ & $121 \pm 38$ & $5.9 \pm 2.5$ \\
$(2,2)$ & $0.178 \pm 0.008$ & $-39 \pm 5$ & $232 \pm 13$ & $43.9 \pm 3.2$ \\
$(3,3)$ & $0.145 \pm 0.005$ & $-1 \pm 8$ & $435 \pm 19$ & $67.0 \pm 3.7$ \\
$(4,4)$ & $0.085 \pm 0.006$ & $-18 \pm 10$ & $285 \pm 25$ & $25.9 \pm 2.9$ \\
$(5,5)$ & $0.072 \pm 0.008$ & $45 \pm 18$ & $327 \pm 45$ & $25.1 \pm 4.4$ \\
$(6,6)$ & $0.53 \pm 0.016$ & $20 \pm 55$ & $268 \pm 56$ & $15.1 \pm 5.6$ \\
\hline $\mathrm{OH} \mathrm{F}=4-4$ & $0.086 \pm 0.006$ & $-13 \pm 10$ & $292 \pm 29$ & $26.7 \pm 3.2$ \\
\hline \multicolumn{5}{c}{ GBT } \\
& $0.136 \pm 0.004$ & $-35 \pm 10$ & $230 \pm 22$ & $33.3 \pm 3.3$ \\
\hline$(1,1)$ & $0.094 \pm 0.003$ & $-42 \pm 10$ & $258 \pm 22$ & $25.8 \pm 2.4$ \\
\hline$(3,2)$ & & & & \\
\hline
\end{tabular}


Table 3. Densities of the molecular gas. The center column is derived using an excitation temperature of $50 \mathrm{~K}$ and the right column using $T_{\text {ex }}=10 \mathrm{~K}$. Whereas $\sum_{\mathrm{J}=1 \ldots 6} N(J, J)$ denotes the sum of the measured lines, the ammonia column densities listed as $\sum_{\mathrm{J}=0 \ldots .6} N(J, J)$ include the $(0,0)$ inversion state that was extrapolated using $T_{\text {rot }}=124 \mathrm{~K}$. The column densities transform into volume densities $n_{\mathrm{NH}_{3} ; \mathrm{J}=0 \ldots 6}$ for an assumed line of sight of $700 \mathrm{pc}$. Finally, for a fractional abundance of $\left[N\left(\mathrm{NH}_{3}\right) / N\left(\mathrm{H}_{2}\right)\right]=10^{-8}$, the resulting molecular hydrogen densities $n_{\mathrm{H}_{2}}$ are shown in the last row. $f_{\mathrm{V}}$ is the volume filling factor.

\begin{tabular}{|c|c|c|}
\hline & $T_{\mathrm{ex}}=50 \mathrm{~K}$ & $T_{\mathrm{ex}}=10 \mathrm{~K}$ \\
\hline & \multicolumn{2}{|c|}{$N\left[10^{15} \mathrm{~cm}^{-2}\right]$} \\
\hline $\mathrm{NH}_{3}(1,1)$ & $3.7 \pm 1.6$ & $0.7 \pm 0.3$ \\
\hline $\mathrm{NH}_{3}(2,2)$ & $21.0 \pm 1.5$ & $4.2 \pm 0.3$ \\
\hline $\mathrm{NH}_{3}(3,3)$ & $28.3 \pm 1.6$ & $5.6 \pm 0.3$ \\
\hline $\mathrm{NH}_{3}(4,4)$ & $10.1 \pm 1.1$ & $2.0 \pm 0.2$ \\
\hline $\mathrm{NH}_{3}(5,5)$ & $9.3 \pm 1.6$ & $1.9 \pm 0.3$ \\
\hline $\mathrm{NH}_{3}(6,6)$ & $5.3 \pm 2.0$ & $1.1 \pm 0.4$ \\
\hline $\mathrm{NH}_{3}(0,0)_{\text {extr }}$ & $6.0 \pm 0.9$ & $1.2 \pm 0.2$ \\
\hline$\sum_{\mathrm{J}=1 \ldots 6} N(J, J)$ & $78 \pm 4$ & $15.5 \pm 0.9$ \\
\hline \multirow{5}{*}{$\sum_{\mathrm{J}=0 \ldots 6} N(J, J)$} & $84 \pm 5$ & $16.7 \pm 1.0$ \\
\hline & $f_{\mathrm{V}}^{-0.5} \times n_{\mathrm{NH}}$ & $\ldots 6\left[10^{-5} \mathrm{~cm}^{-3}\right.$ \\
\hline & $3.9 \pm 0.2$ & $0.8 \pm 0.1$ \\
\hline & $f_{\mathrm{V}}^{-0.5} \times$ & {$\left[10^{3} \mathrm{~cm}^{-3}\right]$} \\
\hline & $3.9 \pm 0.2$ & $0.8 \pm 0.1$ \\
\hline
\end{tabular}

